\title{
EVALUATION AND MODELLING OF UHF RADIOWAVE PROPAGATION IN A FORESTED ENVIRONMENT
}

\author{
Yogita Yadav*
}

\section{*Corresponding Author: -}

\begin{abstract}
: -
Wireless network optimal performance is a major interest in communication engineering. Radio wave propagation in forested environment has been the interest of much theoretical and experimental research over the years. One of the concepts is to use wireless empirical models to predict wireless link quality of service such as path loss and the received power in a transmission domain with irregular terrain. Measurement results of signal strength in UHF band obtained during the two prominent seasons; raining season (when the trees are in leaf) and dry season (when the trees are relatively out of leaf) Idanre-Apomu axis of Ondo State Nigeria were validated against theoretical estimations. However, using the least squared error fit for several sets of measurement data, an empirical model was developed and incorporated into Matlab graphical user interface (GUI) which can be deployed by wireless communication network providers in wireless networks design for path loss prediction and received power in a forested environment.
\end{abstract}

Keywords: Forested environment, measurements, empirical model, UHF band, Matlab GUI.

\section{(c) $(\$)$}




\section{INTRODUCTION}

In radio wave propagation, an interaction between waves and environment attenuates the signal level. It causes path loss and finally limits coverage area. Path loss prediction is a crucial element in the first step of network planning [1]. In order to predict, simulate, and design high- performance communication systems, accurate propagation characteristics of the complex environment have to be known. One of the well-known complex environments is the forest. The appearance of the foliage medium in the path of the communication link has significant effects on the quality of the received signal. This is because, discrete scatterers in the forest such as the randomly distributed leaves, twigs, branches and tree trunks can cause attenuation, scattering, diffraction, and absorption of the radiated propagating waves. This will severely constrain the design of communication systems, and therefore has been of interest to researchers for many years [2]. Since the 1960s, a significant amount of work has been done to investigate the radio wave propagation in forest environment. Both analytical and empirical works on the modeling and characterization of the forested channel have been carried out. Some useful and significant results and analysis are reported in [3]. It is reported that the foliage medium can attenuate the propagating radio wave significantly. There are many external factors that will cause the variation in radio wave propagation and even the complete breakdown of communication link in the forest. Analytical $[4,5]$ and experimental $[6$, 7] work have been performed. However, there is still a significant amount of research work that needs to be performed, especially for the empirical work [6,7] which is site-specific, and limits the practical application of the existing research work. In order to build a robust system that operates well in dense foliage environment, the implementation of MIMO and UWB techniques is often examined. These techniques provide a potential solution to the implementation of a reliable wireless sensor network. However, the successful employment of these techniques in forest environment requires detail knowledge of the effects of the foliage medium on the propagating radio waves.

\section{THEORETICAL BACKGROUND}

In radio wave propagation, the free space path loss model shown in equation 1 acts as a lower bound for the estimation of path loss [8]

Where $\mathrm{f}$ is the frequency in $\mathrm{MHz}, \mathrm{d}$ is the distance between the isotropic transmit and receive antennas in meters. When the radio wave propagates near the ground with a line of sight (LOS) condition, the path loss can be better described by the plane earth (PE) path loss model [8] rather than the free space model. The plane earth path loss model includes the effect of ground reflection and is given as

Where $\mathrm{d}$ is the distance between the isotropic transmit and receive antenna in meters, $\mathrm{hT}$ and hR are the transmit and receive antenna heights, respectively, also in meters. In this model, there is an assumption that $d$ is much larger than the hT and hR.

\section{RADIO WAVE PROPAGATION IN THE FOREST}

Radio waves propagating in the forest naturally experiences multiple scattering, diffraction, and absorption of radiation. These different propagation mechanisms, when combined, can result in severe fades in the received signal, and produce an excess vegetation induced loss as compared to terrestrial propagation. These fade effects have to be considered in order to establish a highly reliable near ground communication link. As a supplement to the well-established theoretical studies [9]- [14] of radio wave behavior in the forest environment, some significant experimental works [15] have been where R0 is the radiation resistance of the dipole antenna in free space and $\mathrm{R}$ is the total antenna resistance in the vicinity of the ground and foliage. Fs is the shadow factor that accounts for the effect of the curvature of the earth, $\mathrm{Fj}$ is the factor that accounts for the effect of the jungle, and hT and hR are the transmit and receive antenna heights, respectively. The relationship between the antenna height and the path loss in equation 3 is verified in [14] through measurements performed within a tropical jungle with foliage depth of up to $6.4 \mathrm{~km}$ in Thailand. In the experiment, horizontally polarized antennas were used at a frequency of $100 \mathrm{MHz}$, with the transmit antenna height, kept at a constant of $24.2 \mathrm{~m}$, and the receive antenna height, varied from 5$30 \mathrm{~m}$. Later, in 1984, Tewari et al. [16] performed an in-depth empirical modeling of antenna height gain on the path loss in the forest, based on the measurements conducted in various tropical rainforest, with foliage depths of up to $4 \mathrm{~km}$ in India. Both vertically and horizontally polarized antennas at frequencies from 50 to $800 \mathrm{MHz}$ were used with the transmit antenna height hT, varying from 3.95-16.45 m, and the receive antenna height $\mathrm{hR}$, varying from $1.5-3.5 \mathrm{~m}$ above the ground, while maintaining the condition of $\mathrm{hT}$. $\mathrm{hR}>10$. They then derived the antenna height gain on the path loss in the forest as shown in equation 4

Fig 1: Schematic Diagram of the Horizontal Foliage Path. ii. Slant path as shown in Fig. 2; the elevation angle is usually above 100 and short foliage path through 1 or 2 trees. These result in different methodologies in the modeling of the foliage-induced loss. But horizontal path is the interest of this study.

Comparatively equations 3 and 4 with the plane earth path loss model in equation 2 shows that, the ground reflected wave has a predominant effect on VHF and UHF radio wave propagation over large foliage depths. This ground reflected wave tends to cancel the direct wave and results in the received field strength (path loss) being proportional (inverse proportional) to the product of the antenna heights for a fixed foliage depth. This reflection effect will be more apparent when the antenna height is low, i.e., near ground. 


\section{AN OVERVIEW OF THE EMPIRICAL FOLIAGE LOSS MODELS}

Much attention has been put into the empirical modeling of the foliage induced excess loss at different frequencies and geometries [17]-[20]. The following summarizes these well-known empirical foliage loss models, which will be discussed and evaluated in this study of the near ground forested radio wave propagation. Based on the ray geometry of the propagating wave, the

The proposed empirical foliage loss models for the horizontal propagation path can be classified as the modified exponential decay (MED) models, such as Weissberger model [21], ITU Recommendation (ITU-R) model [22], COST235 model [23] and fitted ITU-R (FITU-R) model [24]; the modified gradient model, such as Maximum attenuation (MA) model [25], Nonzero gradient (NZG) model [25], and Dual Gradient (DG) model [26]. These models are summarized in Table 1 for reference, and the review of comparative studies among these models is the focus of this subsection. The exponential decay model was first proposed by Weiss- berger [21], and its main modified versions include ITU- R model [22], COST235 model [23] and FITU-R model [24] as shown in Table 1. In general, the exponential decay model has the following form, Where A, B, and C are the fitted parameters from a variety of experiments with regression techniques

\section{RESEARCH METHODOLOGY}

Idanre-Apomu axis in Ondo State Nigeria is a neighbouring town of Akure where the base station is sited. Being a tropical region, the town has a large share of non-uniformly distributed tall trees. The trees of this dense forest can be categorized into three: The tallest trees are distinguishable through their individuality and often about $45 \mathrm{~m}$ in height; next to these are trees between $23 \mathrm{~m}$ and $3.6 \mathrm{~m}$ tall, whose branches extend to one another thereby forming quasi expansive canopy; while the last and most common species of trees in this area are of hard wood. These trees combine with those in others to form impenetrable forest [28]. Signal field strength measurements were carried out in two seasons: wet season in the month of August and dry season in the month of December using a professional TV signal field strength meter type UNAOHM model EP742A. A Yagi array receiving antennae covering both VHF and UHF frequency bands was used for measurements. This was mounted on support about eight meters above the ground to prevent grounding adverse effect on the reception. The Yagi array was coupled through a 50-ohm feeder to a UNAOHM TV strength meter type EP742A, designed for

\section{RESULTS AND DISCUSSION}

The UHF broadcast signal strength measured was compared to the theoretical signal field strength calculated using equation 6 and the result is as shown in

\section{CONCLUSION}

In this research paper, UHF band radiowave propagation in a forested environment was investigated for the two prominent seasons in Nigeria. It is found that, many factors such as antenna heights; depolarization etc. can affect the radio wave propagation within a forested channel. However, a wireless Matlab based GUI empirical model was developed for path loss prediction and received power which are the major factors in wireless network planning in communication system. Results from this research work are useful for the planning of a reliable communication link in the forest environments.

\section{REFERENCES}

[1].Famoriji J. Oluwole and Olasoji Y. Olajide, "Radio Frequency Propagation Mechanisms and Empirical Models for Hilly Areas", Canadian Journal on Electrical and Electronics Engineering, Vol. 4, No. 2, April 2013, PP. 65- 70.

[2].Meng Y. S., Lee Y. H. and Hg B. C. "Study of Propagation

[3].Loss Prediction in Forested Environment" Progress in Electromagnetic Research B, Vol. 17, 2009, PP. $117-133$.

[4].Bertoni, H. L., Radio Propagation for Modern Wireless Systems, Prentice Hall PTR, New Jersey, 2000.

[5].Liao, D. and K. Sarabandi, INear-earth wave propagation characteristics of electric dipole in presence of vegetation or snow layer," IEEE Trans. Antennas Propag., Vol. 53, No. 11, 3747\{3756, 2005.

[6].Liao, D. and K. Sarabandi, "Modeling and simulation of near- earth propagation in presence of a truncated vegetation layer", IEEE Trans. Antennas Propag., Vol. 55, No. 3, 949\{957, 2007.

[7].Joshi, G. G., C. B. Dietrich, C. R. Anderson, W. G. Newhall, W. A. Davis, J. Isaacs, and G. Barnett, "Near- ground channel measurements over line-of-sight and forested paths”, IEE Proc. Microw. Antennas Propag., Vol. 152, No. 6, $589\{596,2005$.

[8].Anderson, C. R., H. I. Volos, W. C. Headley, F. C. B. Muller, 130 Meng, Lee, and Ng and R. M. Buehrer, ULow antenna ultra-wideband propagation measurements and modeling in a forest environment," Proc. IEEE Wireless Commun. Networking Conf., 1229\{1234, Las Vegas, USA, 2008.

[9].Parsons J. D., The Mobile Radio Propagation Channel, 2nd ed. New York: Wiley, 2000. 